that both hemispheres share the "word-hearing" function; the right one, however, to a less degree than the left.

Among the other cases was one of Dr. Milligan's, in which, in the after-treatment of the radical operation, no packing had been used, and yet a good result was obtained by means of skin-grafting eight days after the operation. A diminution in energy in the use of packing is a healthy sign, as excess of zeal in this direction sometimes defeats its own object. Dr. Tilley referred to a colleague's method of using installations of alcohol at a very early stage in the after-treatment of these cases-a method much in rogue at the Central London Throat and Ear Hospital, where it was introduced by $\mathrm{Mr}$. Heath. One of the most instructive cases was one brought forward by Dr. Permewan, in which caries behind the posterior border of the mastoid, associated with middle-ear disease, developed without perforation of the membrane. The author was led to its discovery by the formation of an abscess over the mastoid; he opened this and found a cavity from which pus exuded, and which communicated with the posterior fossa of the skull; the opening was $\frac{3}{4}$ inch behind the auditory meatus. The case shows the importance of adopting careful aseptic measures in opening all abscesses in this neighbourhood, however superficial they may seem to be. The happy result in Dr. Permewan's case was no doubt due to his caution in this respect.

Owing to illness, the President, Dr. Crban Pritchard, was prevented from presiding; but Mr. Ballance took the chair for the commencement of the meeting, and Mr. Hugh Jones for the later part.

We are sure our readers will peruse the reports of this meeting with profit and pleasure.

\title{
HUGH E. JONES'S LANDMARK FOR THE FACIAL NERVE.
}

Is an interesting and timely communication, read at a recent meeting of the Otological Society of the United Kingdom, Mr. Hugh E. Jones described a landmark for guidance during the performance of the radical mastoid operation, which, whether or not it be universally applicable, has, at all events, the merit of being readily available during the performance of the operation, and therefore of the utmost practical value.

It is familiar to all that the facial nerve is embedded to a certain extent in the lowest part of the innermost section of the posterior wall of the external auditory meatus, and it would therefore seem 
natural to suppose that the descending portion of the aqueduct of Fallopius ran downwards and outwards with a considerable amount of obliquity. This, however, is more apparent than real, and, in fact, the direction is almost absolutely vertical. This is well shown in our abstract of an article by Dr. Randall of Philadelphia (see this Journal, p. 335). Professor Schwartze (vide abstract, Jorrsal of LarYngologx, May, 1903, p. 277) gives his opinion that there is a certain possible variation between the extremes of verticality and obliquity respectively; but this is not very considerable, and the apparent obliquity depends upon the position of the plane of the tympanic ring, which, of course, lies much deeper at its lower than at its upper part. Mr. Hugh Jones points out that the part of the anditory canal in uhich the facial nerre is embedded is entirely internal to the highest part of the ftror of the meatus, and below a line running from this upwards and inwards at right angles to the plane of the tympanic ring. We may, therefore, chisel away with confidence all the posterior osseous wall lying external and superior to this line. Nany important measurements have been made and published by observers of authority, but they are difficult of application during operation, and practical surgeons will value this readily employed landmark, for which we are indebted to Mr. Hugh. E. Jones.

D. G.

\section{ON THE TRANSILLUMINATION OF THE MAXILLARY ANTRUM AND OF THE EYE.}

By C. Zien, M.D. (Danzig).

(Translated hy A.rThosy IICCALI, M.D.)

THE remark made by Lennox Browne that it requires, as a rule, twenty years for a new truth to become known also holds good for the persistence of many errors, and appears to apply in a special degree to the exaggerated importance which has been attached to the transillumination of the maxillary antrum in the diagnosis of cases of suppuration, as is shown by a glance at the most recent literature in rhinology and ophthalmology. In order, therefore, to assist in giving the quietus to transillumination in this connection, I should like to report some experiments made on patients in $\mathrm{my}$ own practice, and also corresponding experiments on animals.

The untrustworthiness of both the Heryng-Yohsen symptom (that is, the difference shown on transilluminating the cheeks) and the Davidsohn symptom (that is, the difference in the illumination 